Research Article

\title{
Study on the Miscibility of Polypyrrole and Polyaniline Polymer Blends
}

\author{
Tekalign A. Tikish $\mathbb{D}^{1,2,3}$ Ashok Kumar, ${ }^{2}$ and Jung Yong Kim ${ }^{1,4}$ \\ ${ }^{1}$ School of Materials Science and Engineering, Jimma Institute Technology, Jimma University, P.O. Box 378, Jimma, Ethiopia \\ ${ }^{2}$ Department of Physics, Tezpur University, Napaam, Sonitpur, Assam 784028, India \\ ${ }^{3}$ Emerging Technology Centre, Materials Science Directorate, Ethiopian Biotechnology Institute, P.O. Box 5954, \\ Addis Ababa, Ethiopia \\ ${ }^{4}$ School of Chemical Engineering, Jimma Institute Technology, Jimma University, P.O. Box 378, Jimma, Ethiopia
}

Correspondence should be addressed to Tekalign A. Tikish; tekisht@gmail.com

Received 2 April 2018; Revised 7 June 2018; Accepted 20 June 2018; Published 19 August 2018

Academic Editor: Yahya A. Ismail

Copyright (C) 2018 Tekalign A. Tikish et al. This is an open access article distributed under the Creative Commons Attribution License, which permits unrestricted use, distribution, and reproduction in any medium, provided the original work is properly cited.

We report on the miscibility and phase behaviour of polypyrrole-polyaniline (PPy/PANI) as a function of blend composition. The $\mathrm{PPy} / \mathrm{PANI}$ blends were prepared by solution processing method, using dimethyl sulfoxide (DMSO) solvent. Characterization of the polymer blends was carried out based on the data analysis from Fourier transform infrared spectroscopy (FT-IR), X-ray diffraction (XRD), and differential scanning calorimetry (DSC). The PPy/PANI system was successfully formed blends in DMSO solvent. The polymer blends showed almost amorphous nature in XRD spectra because of intermolecular interaction between PPy and PANI macromolecules, which was confirmed by FT-IR data. Specifically, the DSC result for the PPY : PANI $=50: 50$ wt. $\%$ blend showed only one glass transition temperature $\left(T_{\mathrm{g}}\right)$, which indicates that the two polymers are well miscible without undergoing any phase separation.

\section{Introduction}

Recently, polymer blends have attracted many scientists to devote their lives to study the polymer-polymer system in order to develop new functional plastics with technologically attractive properties [1]. Hence, the polymer blend has been one of the hot research areas for material scientists and engineers because of its versatile applications [2]. Specifically, conjugated polymer blend system is not an exception for this trend of attraction. So far, conjugated polymers showing metallic or semiconducting properties have been commonly synthesized through electrochemical and chemical oxidative polymerization [3]. Between these two methods, the former electrochemical methods produce a film with brittle and not processable property [4]. However, the latter method, that is, chemical oxidative polymerization provides a better opportunity for producing significantly a solution processable polymer, compared to the electrochemical methods. Thus, many efforts have been made to further improve the processability by preparing soluble conducting polymer through chemical oxidative method, for example, grafting alky side chains to polymeric backbone, and so on. [5]. The other approach to improve the processability of conducting polymer is to blend it with another material such as saturated hydrocarbon or $\pi$-conjugated semiconducting polymer [6]. Since mid-1980s, the blending of polyaniline (PANI) with other soluble insulating polymers has received great attention for its potential application to a wide variety of optical and electronic devices [7]. For example, the blending of PANI with polypyrrole (PPy) has enhanced the performance of materials, based on improved mechanical, thermal, electrical, and other processing properties [8]. The recent approaches for preparing polymer semiconductors are through solution processing methods. This method is advantageous from various points of view, such as cost minimization, scale-up, and roll-to-roll 


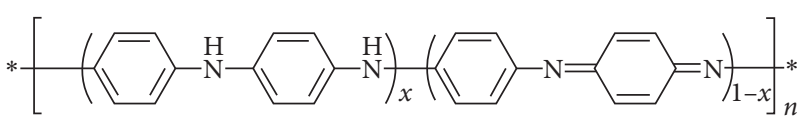

(a)

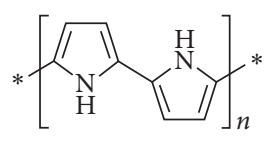

(b)

FIgURe 1: Chemical structure of polyaniline (PANI) and polypyrrole (PPy).

processing [9]. The variety of functional chain molecules can be blended with other materials for fine-tuning device performance. Specifically, this blending approach is extremely common in organic photovoltaics (OPV) or organic field-effect transistor (FET) when they prepare active layer or semiconducting channel layer, respectively [10]. In line with this blend approach, the conjugated polymer such as PPy or PANI also has been compounded with various nanomaterials, nanoparticles, and other organic materials for improving materials' properties for advanced applications. Figure 1 shows their chemical structures.

To date, some amounts of researches have been carried out for characterizing the PANI/PPy composites and/or blends. Xing et al. prepared PANI/PPy composite from a PANI dispersion using sodium dedocylbenzene sulfonate (SDBS) as both dopant and surfactant in $\mathrm{HCl}$ acid. And they reported that the $\mathrm{PANI} / \mathrm{PPy}$ system has exhibited some specific interactions between PANI and PPy macromolecules. Also, they pointed out that their PANI/PPy composite has high conductivity based on excellent sponge-like morphologies [11]. According to another research using copolymer such as poly(aniline-co-pyrrole) (PAP), some nanostructures were constructed for application to chemiresistive ammonia sensor, in which the copolymer PAP sensor showed improved sensing ability towards low concentration of ammonia, as compared to its homopolymer PANI or PPy sensors at room temperature. The PAP sensor was reported to be very reliable in terms of cost, recovery time, selectivity, reproducibility, detection range, and stability [12]. The next work indicated that highly conductive PAP copolymer was synthesized by properly controlling prepolymerization time, which showed improved conductivity without any significant change of morphologies of a film [13]. Akundy et al. used electrochemical deposition technique for synthesis of a PANI/PPy composite on the aluminium substrates in the aqueous oxalic acid solution. They reported that some morphology changes were observed in their PANI/PPy composite [14]. The aforementioned results are some background works for studying on the PANI/PPy blend or nanocomposite systems.

In general, there are three common categories of synthesis of PANI/PPy blends or composites, which are (a) electrochemical deposition, (b) copolymerization, and (c) solution processing [15]. They are used in drug delivery, encapsulation, supercapacitor, sensor, and other optoelectronic/electronic devices such as solar cells, light emitting diode, and FET [2]. However, the PANI/PPy system has only a limited application because of its partial miscibility or solubility in the common organic solvents. This limitation affects all optical and electrical properties because of unfavourable processability. Hence, the enhancement of processability through enhanced miscibility of the PANI/PPy system is very desirable [16].

In thermodynamics, miscibility or solubility means mixing of two or more components on the molecular level, which results in a homogeneous structure with equal thermodynamic state variables. For mixing, the Gibbs free energy of mixing $\left(\Delta G_{\text {mix }}\right)$ must be negative for forming a homogeneous system. According to the principles of thermodynamics, when $\Delta G_{\text {mix }}$ is negative, the thermodynamic miscibility and homogeneity can be reached. This condition can be fulfilled in the case when strong specific intermolecular interactions are located between the components of a polymer blend. Of course, sometimes, we may have a case, in which there is only moderate or poor interaction, inducing a partial miscibility as a function of temperature and composition [17]. At this moment, note that one way to examine the miscibility and preliminary affinity between two polymers is to study the change in the glass transition temperature $\left(T_{\mathrm{g}}\right)$ of polymers after mixing. If the mixture exhibits a single $T_{\mathrm{g}}$, this evidence means that the two homopolymers are usually miscible. However, oppositely, if the mixture displays more than one $T_{\mathrm{g}}$, the system is believed to be partially miscible or immiscible [18]. Based on these understandings, in this study, we report the miscibility and phase behaviour of the PANI/PPy system as a function of blending composition, for which FT-IR, XRD, and DSC were utilized.

\section{Materials and Methods}

Polyaniline was synthesized by chemical oxidative polymerization of aniline in the presence of hydrochloric acid and ammonium persulfate. A $1 \mathrm{M}$ of aniline was dissolved in hydrochloride solution. Then, $0.15 \mathrm{M}$ ammonium persulfate (APS) was dissolved in $50 \mathrm{ml}$ of double-distilled water. After that, we added the APS solution dropwise to hydrochloride solution to initiate the polymerization of aniline. The mixture was stirred with magnetic stirrer for $9 \mathrm{~h}$ under $5^{\circ} \mathrm{C}$ temperature. Then, we added methanol to stop further polymerization and let it unagitated until being precipitated. The resulting precipitate was collected by filtration. Then, the product was washed successively by $0.2 \mathrm{M}$ of hydrochloride, double-distilled water, and acetone, sequentially. Through washing the samples with hydrochloride, we can remove any undesirable residual monomer, oxidant, and other decomposed products. And also, we provide uniform protonation to PANI and wash the sample again by acetone for removing low-molecular-weight organic intermediates and oligomers. Then, finally, our product was obtained as a fine powder, which was dried under vacuum at $50^{\circ} \mathrm{C}$ for $24 \mathrm{~h}$.

Polypyrrole was synthesized by chemical oxidative polymerization of pyrrole. The $1 \mathrm{M}$ of pyrrole was dissolved in 
double-distilled water $(10 \mathrm{ml})$. Then, $1.622 \mathrm{~g}$ of $\mathrm{Fe}_{2} \mathrm{Cl}_{3}$ was dissolved into $50 \mathrm{ml}$ of double-distilled water. After that, the reacting monomers were mixed slowly in a dropwise manner under constant stirring for 30 minutes at $5^{\circ} \mathrm{C}$. After that, the polymerization was allowed for $6 \mathrm{~h}$ under stirring. Then, we kept it unagitated for $24 \mathrm{~h}$ until PPy powders were settled down. The resulting precipitate was collected by filtration. And the products were washed by double-distilled water many times and then dried in oven for $12 \mathrm{~h}$ at $50^{\circ} \mathrm{C}$.

The PANI/PPy samples were prepared by the solution processing method. The PPy polymer was blended with PANI in various compositions, such as PPy: PANI $=50: 50$, $70: 30,80: 20$, and $90: 10$ in wt.\%, for which the solvent DMSO was used. As a first step, the PPy and PANI solutions were prepared, respectively, in which ca. $20 \mathrm{mg}$ of polymer was dissolved in $10 \mathrm{ml}$ of DMSO, and the mixture was ultrasonicated for $10 \mathrm{~min}$. Then, the two individual solutions were mixed together according to desirable weight ratio and again ultrasonicated for additional $1 \mathrm{~h}$. Finally, the solvent used was evaporated in an oven at $50^{\circ} \mathrm{C}$ for $12 \mathrm{~h}$ for obtaining our PANI/PPy samples.

\section{Results and Discussion}

3.1. The FT-IR Characteristics. In Figure 2(a), the FT-IR spectra of PANI samples, the peak observed at $3433.75 \mathrm{~cm}^{-1}$ is due to $\mathrm{N}-\mathrm{H}$ stretching. The absorption bands at 2923.21 and $2825.55 \mathrm{~cm}^{-1}$ are due to asymmetric $\mathrm{C}-\mathrm{H}$ stretching and symmetric $\mathrm{C}-\mathrm{H}$ stretching vibrations, respectively. The absorption peaks observed at $1637.68 \mathrm{~cm}^{-1}$ were attributed to $\mathrm{C}=\mathrm{C}$ stretching in aromatic nuclei. The band obtained at $1600-1500 \mathrm{~cm}^{-1}$ corresponds to $\mathrm{C}-\mathrm{H}$ stretching in aromatic compounds. In Figure 2(b), the spectra of PPy, the strong peak observed at $3447 \mathrm{~cm}^{-1}$ is related to $\mathrm{N}-\mathrm{H}$ stretching. The peak at $2915 \mathrm{~cm}^{-1}$ is related to $\mathrm{CH}_{2}$, and the peak observed at $1536 \mathrm{~cm}^{-1}$ is due to $\mathrm{C}-\mathrm{C}$ and $\mathrm{C}=\mathrm{C}$ stretching in the ring. The peak of $\mathrm{C}-\mathrm{N}$ stretching can be found at $1483 \mathrm{~cm}^{-1}$. The peak $1169 \mathrm{~cm}^{-1}$ refers to C-C PPy ring, while the peaks of $786 \mathrm{~cm}^{-1}$ and $1036 \mathrm{~cm}^{-1}$ can be related to $\mathrm{C}-\mathrm{H}$ out-of-plane bending and in-plane deformation, respectively. The FT-IR spectra for the PPy/PANI system in various compositions are shown in Figures 2(c)-2(f). The spectra of PPy: PANI = 70:30 and PPy: PANI $=90: 10$ on Figures 2(f) and 2(e), respectively, indicated that there is a change of spectra due to the different composition in the PPy/PANI system. The PPy: PANI = 50:50 and PPy $:$ PANI $=80: 20$ spectra on Figures 2(c) and 2(d) showed nearly very trivial change. Overall, the FT-IR spectra showed that there is the change of intermolecular interactions between PPy and PANI with composition, resulting in the change of spectral shape. Specifically, we notice that the specific interactions between PPy and PANI macromolecule were confirmed not only by the change of intensity and shape observed in the range of spectra at $1645-1130 \mathrm{~cm}^{-1}$ for the samples of PANI, PPy, and PPy: PANI $=50: 50$, but also by the peak shift observed around the spectra at $980-786 \mathrm{~cm}^{-1}$. Furthermore, the peak's broadening and shift was observed around $793-648 \mathrm{~cm}^{-1}$. Therefore, we may expect that these intermolecular interactions might allow the PPy/PANI mixture to form blends.

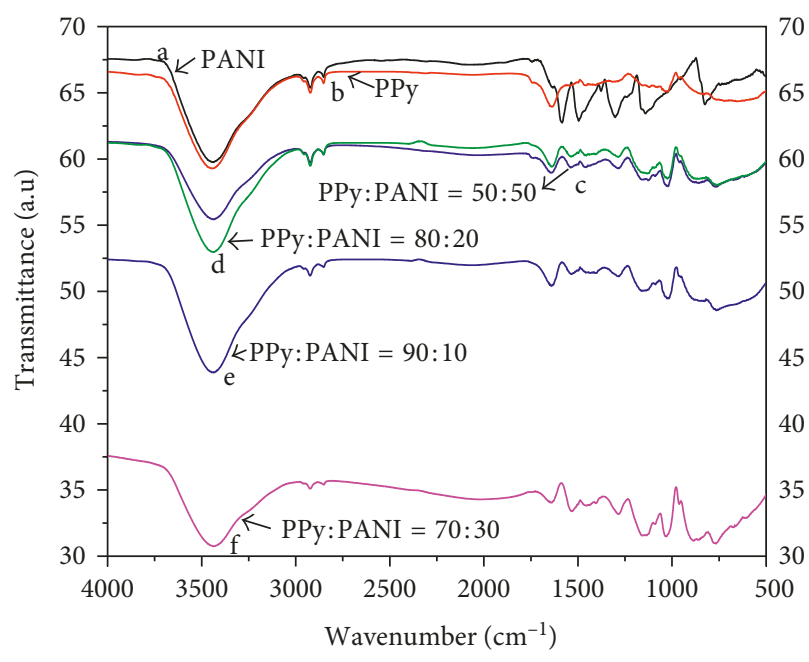

Figure 2: FT-IR spectra of PANI, PPy, the mixture of PPy: $\mathrm{PANI}=50: 50, \mathrm{PPy}: \mathrm{PANI}=70: 30, \mathrm{PPy}: \mathrm{PANI}=80: 20$, and $\mathrm{PPy}:$ PANI $=90: 10($ wt. $\%)$.

3.2. The XRD Characteristics. Figure 3(a) displays the PANI's XRD pattern, in which a broad peak was observed at about $2 \theta=20^{\circ}$ and sharp peak at about $2 \theta=26^{\circ}$. The peak at $26^{\circ}$ is more intensive than the peak at $20^{\circ}$. The peak around $26^{\circ}$ indicates that PANI is a semicrystalline polymer, and the peak around $20^{\circ}$ displays that PANI has an amorphous character also. Figure 3(b) describes pure PPy spectra, in which the broad peaks at around $2 \theta=24^{\circ}$ indicate that PPy has significant amorphous nature compared to PANI. The other two small peaks are observed at $33^{\circ}$ and $35^{\circ}$, indicating chain ordering in PPy macromolecules in spite of major amorphous nature of PPy. Figures 3(c)-3(f) show the XRD spectra of the $\mathrm{PPy} / \mathrm{PANI}$ system as a function of composition, in which we studied the miscibility and crystallinity of the PPy/PANI system. If phase separation occurs in the PPy/PANI system, $\mathrm{X}$-ray diffraction peaks from both PPy and PANI are expected to be observed in the compound. However, if not, the absence of XRD peaks from pure polymer, PANI or PPy, indicates that the PPy/PANI system is in a single phase, confirming a blend system. Figures 3(c) to 3(f) show that the weight percent of PANI in the PPy/PANI system is 50, 30, 20, and 10, respectively. The result pointed out that the XRD spectra of PPy: PANI $=50: 50$ in Figure 3(c) are very distinguished from the other compositions, $70: 30,80: 20$, and $90: 10$, of the system in Figures 3(d)-3(f). In Figures 3(d)-3(f), the diffraction peaks of both pure PPy and PANI are observed, indicating they are composite rather than blend. This result showed that the PPy: PANI systems with $70: 30,80: 20$, and $90: 10$ mixing ratio showed a phase separation; that is, they might be nanocomposites. In contrast, the spectrum of the PPy: $\mathrm{PANI}=50$ : 50 system in Figure 3(c) shows that the peaks at $2 \theta=26^{\circ}$ (from PANI) and the peaks at $2 \theta=26^{\circ}$ (from PPy) are disappeared. This XRD results indicated that in the PPy: PANI $=50: 50$ system, the crystallinity was disappeared through well miscibility (i.e., no phase separation). This observation means that the interactions between PPy and PANI are very high to allow each polymer to mix in a molecular level. Therefore, we confirm that the PPy: PANI = 50:50 (wt.\%) is a polymer 


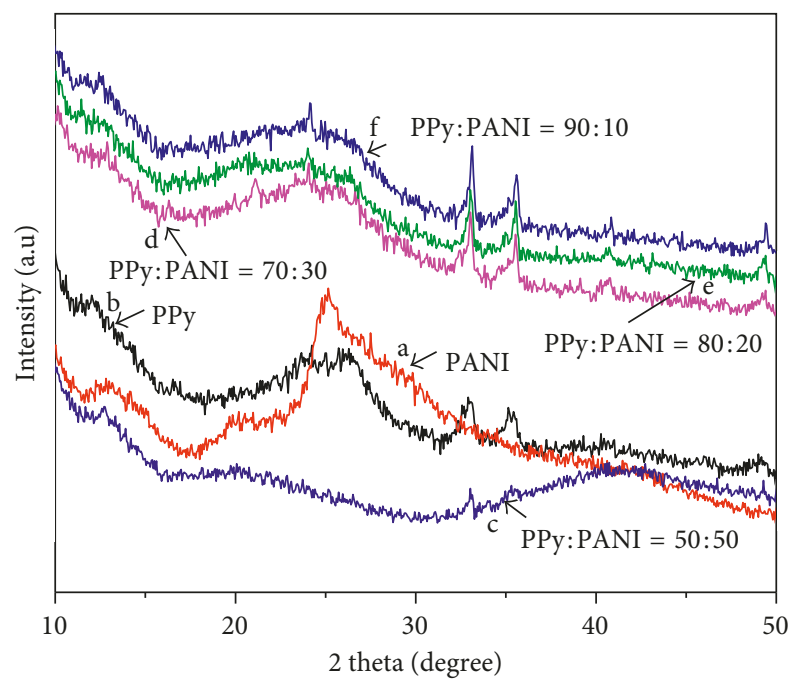

Figure 3: XRD of (a) PANI, (b) PPy, and (c-f) PPy/PANI mixture.

blend instead of composite. Finally, we double-checked its blending/miscibility/single-phase possibility through thermal properties, for example, DSC results.

3.3. The DSC Characteristics. The DSC analysis was done to assess the thermal effect on phase behaviour. In polymer science, the glass transition temperature is significantly important to determine whether a polymer-polymer mixture is miscible or immiscible or partly miscible. In the DSC thermogram, if a single $T_{\mathrm{g}}$ was observed for a mixture, we would say that it a blend with miscibility. However, if the two $T_{\mathrm{g}}$ were observed in a mixture, we would decide that the components are an immiscible system, that is, a composite. Figures 4(a)-4(c) show the glass transition temperatures of pure PANI, pure PPy, and the PPy: PANI = 50:50 mixture, respectively. The DSC curve of PANI and PPy shows broad endothermic peaks at $359.95 \mathrm{~K}$ and $384.85 \mathrm{~K}$, respectively. In literatures, PANI's $T_{\mathrm{g}}$ was observed at $385.15 \mathrm{~K}$ [19], and PPy's $T_{\mathrm{g}}$ was displayed at $371.15 \mathrm{~K}$ [20], indicating that our measured $T_{\mathrm{g}}$ is close to the literature's reports within $\sim 10-20^{\circ} \mathrm{C}$ error. When evaluating the PPy: PANI $=50: 50$ system in Figure 4(c), we observed that there is only single glass transition temperature. This key result indicated that the two polymers, PPy and PANI, are a miscible system when we are blending them with 50:50 weight ratios. The glass transition temperatures of each pure polymer and their PPy: PANI = 50:50 mixture are summarized in Table 1. Note that the $T_{\mathrm{g}}$ of the PPy:PANI $=50: 50$ system is falling between the pure component polymers.

\section{Conclusion}

The PPy/PANI system was successfully obtained by the solution processing method using DMSO solvent. In the XRD spectrum, the PPy: $\mathrm{PANI}=50: 50$ system showed that the peaks originated from pure PPy or PANI disappeared, which indicates that the characteristic of crystallinity disappeared and resultantly the PPy: PANI = 50:50 system has amorphous nature. In the FT-IR analysis, the blending of

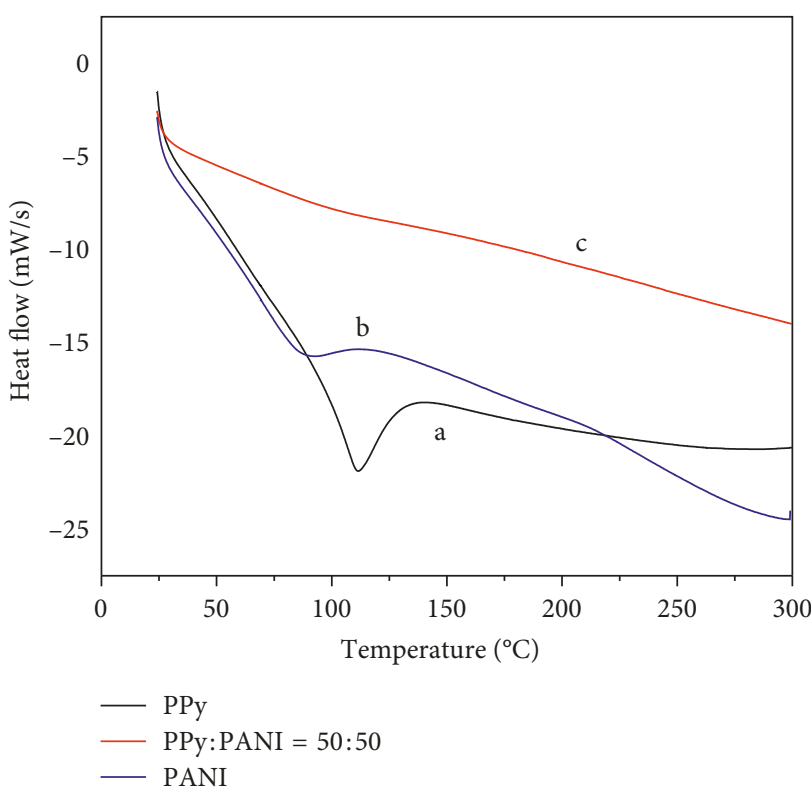

FIgURE 4: The DSC analysis to examine the miscibility of the PPy/PANI system.

TABle 1: Glass transition of PPy, PANI, and PPy: PANI $=50: 50$.

\begin{tabular}{ll}
\hline Polymer mixture $($ wt. $\%)$ & $T_{\mathrm{g}}(\mathrm{K})$ \\
\hline PPy $:$ PANI $=0: 100$ & 359.95 \\
PPy $:$ PANI $=100: 0$ & 384.85 \\
PPy $:$ PANI $=50: 50$ & 375.78 \\
\hline
\end{tabular}

PPy and PANI makes the FT-IR spectra's intensity and shape be changed. The broadening and shift of peaks was also observed. These phenomena confirm that there are specific interactions between PPy and PANI macromolecules when blended together. Finally, the DSC thermogram indicated that there is only single glass transition temperature for the PPy: PANI $=50: 50$ system. Therefore, we conclude that the PPy $:$ PANI $=50: 50$ system is a miscible polymer blend.

\section{Data Availability}

The data that support the findings of this study are available from the corresponding author upon the reasonable request. The data are not publicly due to confidentiality and privacy research participants.

\section{Conflicts of Interest}

The authors declare that they have no conflicts of interest.

\section{Acknowledgments}

The authors are thankful for funds from the Ethiopian Ministry of Education. Also, the authors gratefully acknowledge the Department of Physics, Materials Research Laboratory, Tezpur University, India, and Ethiopian Biotechnology Institute. 


\section{References}

[1] D. Seong, J. Yeo, and S. Wang, "Fabrication of polycarbonate blends with poly(methyl methacrylate-co-phenyl methacrylate) copolymer: miscibility and scratch resistance properties," Journal of Industrial and Engineering Chemistry, vol. 36, pp. 251-254, 2016.

[2] A. Facchetti, " $\pi$-conjugated polymers for organic electronics and photovoltaic cell applications," Chemistry of Materials, vol. 23, no. 3, pp. 733-758, 2011.

[3] T. Le, Y. Kim, and H. Yoon, "Electrical and electrochemical properties of conducting polymers," Polymers, vol. 9, no. 12, p. 150, 2017.

[4] N. S. M. Tahiruddin and R. Daik, "Synthesis and characterization of anionic surfactants doped polypyrrole," Der Pharma Chemica, vol. 7, no. 6, pp. 159-162, 2015.

[5] H. Behniafar and K. Malekshahinezhad, "A unique path to reach thermostable polypyrrole/Pd microfibers via chemical oxidative polymerization," Colloid and Polymer Science, vol. 292, no. 9, pp. 2083-2088, 2014.

[6] X. Wang, L. C. Groff, and J. D. McNeill, "Multiple energy transfer dynamics in blended conjugated polymer nanoparticles," Journal of Physical Chemistry C, vol. 118, no. 44, pp. 25731-25739, 2014.

[7] V. Jousseaume, M. Morsli, A. Bonnet, O. Tesson, and S. Lefrant, "Electrical properties of polyaniline-polystyrene blends above the percolation threshold," Journal of Applied Polymer Science, vol. 67, no. 7, pp. 1205-1208, 1998.

[8] J. Tahalyani, K. K. Rahangdale, and K. Balasubramanian, "The dielectric properties and charge transport mechanism of p-conjugated segments decorated with intrinsic conducting polymer," RSC Advances, vol. 6, no. 74, pp. 69733-69742, 2016.

[9] B. Kang, W. H. Lee, and K. Cho, "Recent advances in organic transistor printing processes," ACS Applied Materials and Interfaces, vol. 5, no. 7, pp. 2302-2315, 2013.

[10] J. Li, Y. Zhao, H. S. Guo et al., "A stable solution-processed polymer semiconductor with record high-mobility for printed transistors," Scientific Reports, vol. 2, no. 1, p. 754, 2012.

[11] S. Xing, C. Zhao, T. Zhou, S. Jing, and Z. Wang, "Preparation and characterization of polyaniline-polypyrrole composite from polyaniline dispersions," Journal of Applied Polymer Science, vol. 104, no. 6, pp. 3523-3529, 2007.

[12] V. Chaudhary and A. Kaur, "Enhanced and selective ammonia sensing behaviour of poly(aniline co-pyrrole) nanospheres chemically oxidative polymerized at low temperature," Journal of Industrial and Engineering Chemistry, vol. 26, pp. 143-148, 2015.

[13] X. Ou and X. Xu, "A simple method to fabricate poly(anilineco-pyrrole) with highly improved electrical conductivity via pre-polymerization," RSC Advances, vol. 6, no. 17, pp. 13780-13785, 2016.

[14] G. S. Akundy, R. Rajagopalan, and J. O. Iroh, "Electrochemical deposition of polyaniline-polypyrrole composite coatings on aluminum," Journal of Applied Polymer Science, vol. 83, no. 9, pp. 1970-1977, 2002.

[15] A. Pud, N. Ogurtsov, A. Korzhenko, and G. Shapoval, "Some aspects of preparation methods and properties of polyaniline blends and composites with organic polymers," Progress in Polymer Science, vol. 28, no. 12, pp. 1701-1753, 2003.

[16] N. Su, "Improving electrical conductivity, thermal stability, and solubility of polyaniline-polypyrrole nanocomposite by doping with anionic spherical polyelectrolyte brushes," Nanoscale Research Letters, vol. 10, no. 1, p. 301, 2015.
[17] H. W. Milliman, D. Boris, and D. A. Schiraldi, "Experimental determination of Hansen solubility parameters for select POSS and polymer compounds as a guide to POSS-polymer interaction potentials," Macromolecules, vol. 45, no. 4, pp. 1931-1936, 2012.

[18] F. E. Földes, F. Damsits1, E. Fekete, and B. Pukánszky, "Interaction-structure-property relationships in amorphous polymer blends," Polymer Bulletin, vol. 44, no. 4, pp. 363-370, 2000.

[19] M. T. Ramesan and T. Sampreeth, "In situ synthesis of polyaniline/Sm-doped $\mathrm{TiO}_{2}$ nanocomposites: evaluation of structural, morphological, conductivity studies and gas sensing applications," Journal of Materials Science: Materials in Electronics, vol. 29, no. 5, pp. 4301-4311, 2018.

[20] M. T. Ramesan and V. Santhi, "In situ synthesis, characterization, conductivity studies of polypyrrole/silver doped zinc oxide nanocomposites and their application for ammonia gas sensing," Journal of Materials Science: Materials in Electronics, vol. 28, no. 24, pp. 18804-18814, 2017. 


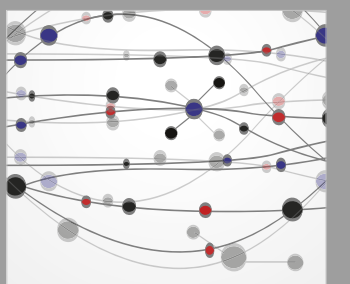

The Scientific World Journal
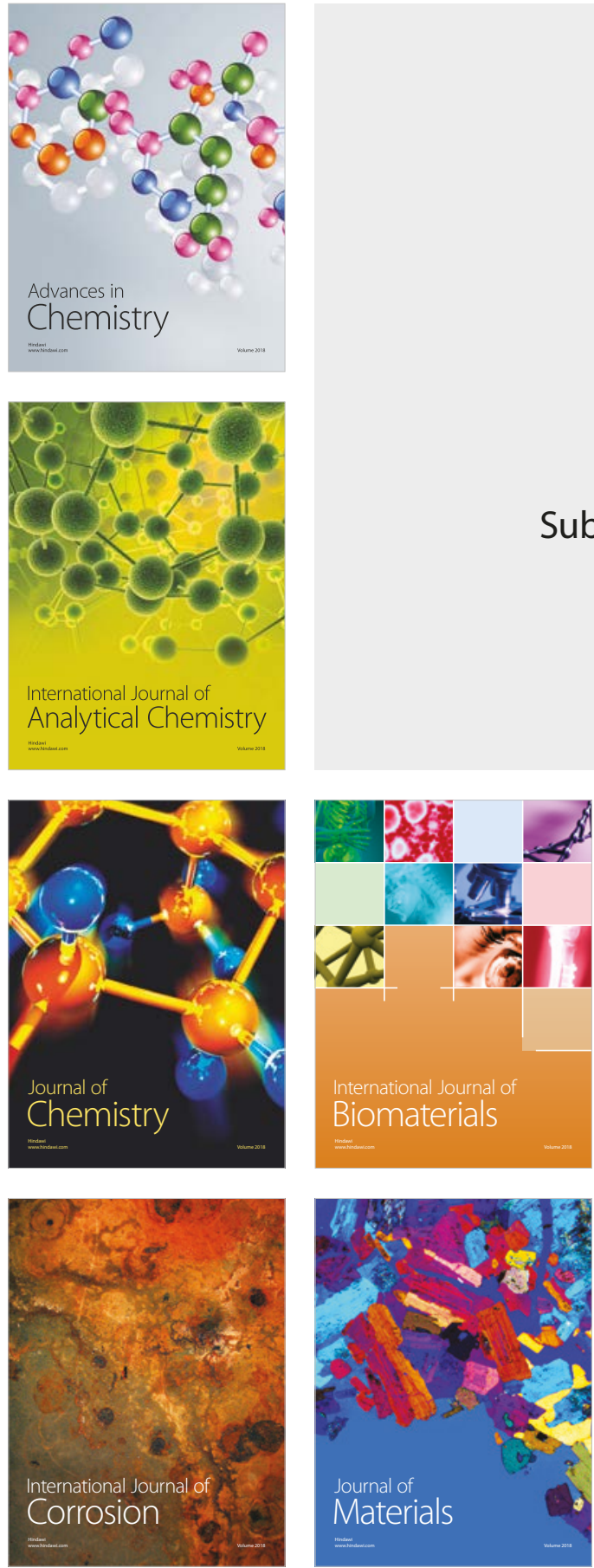

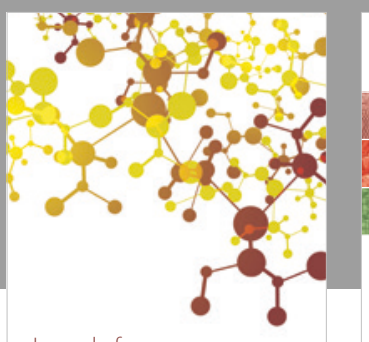

Journal of

Applied Chemistry
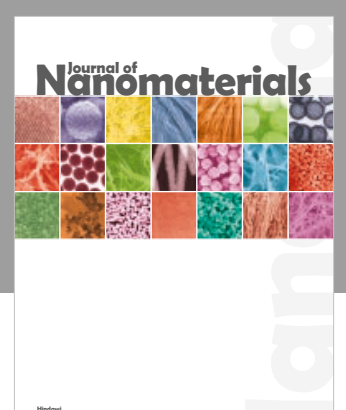

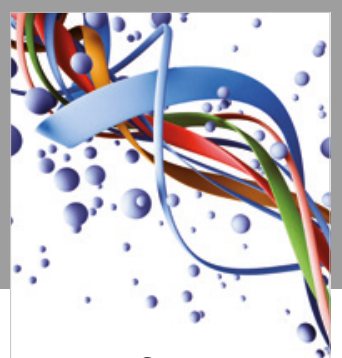

Scientifica

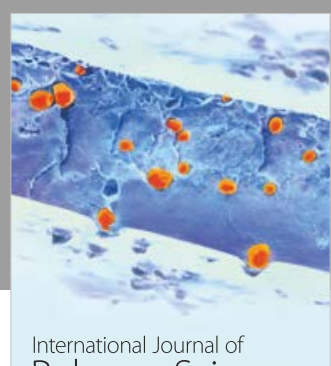

Polymer Science

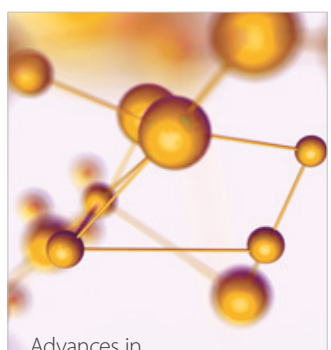

Physical Chemistry
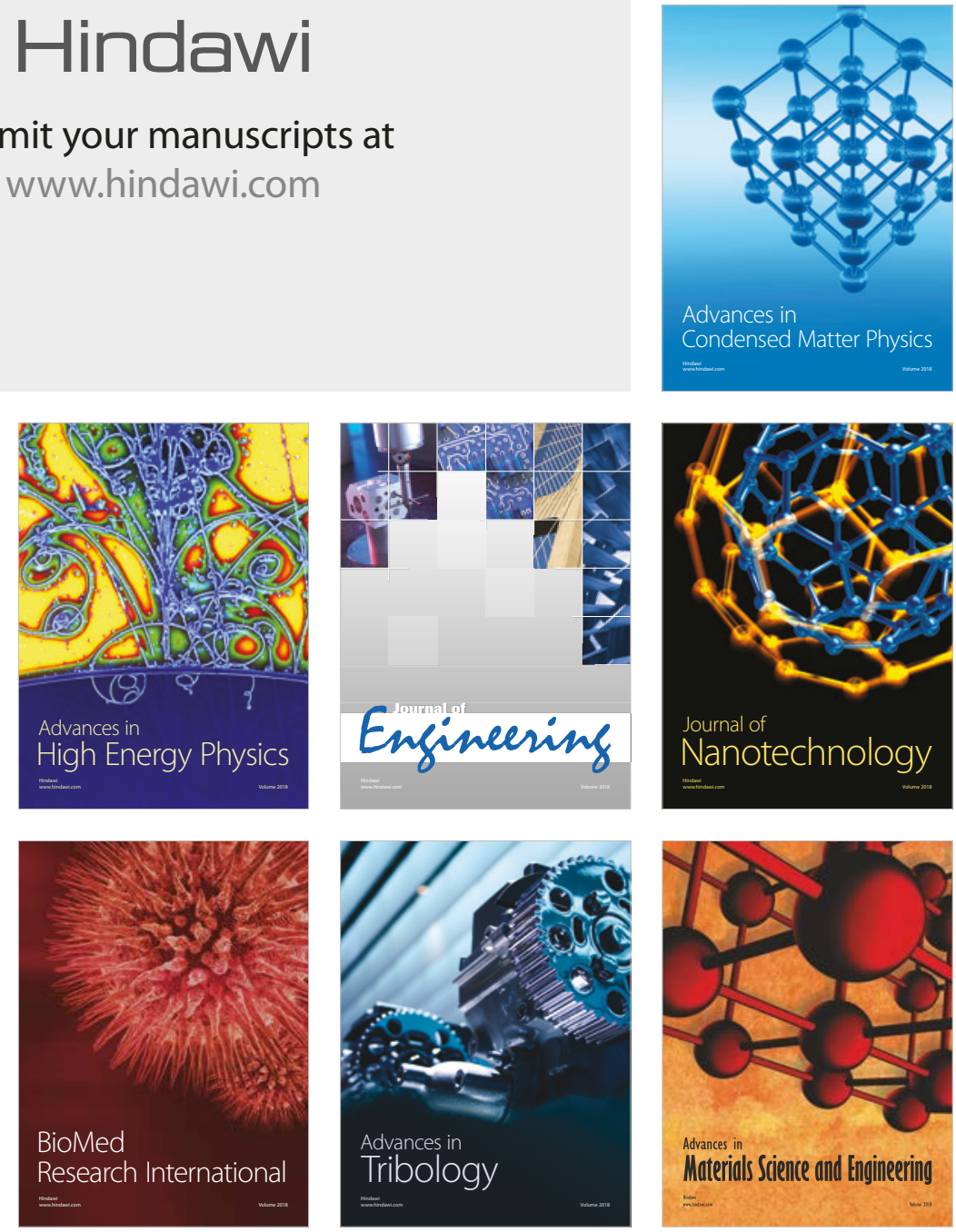